\title{
Transition to hydrodynamics in colliding fermion clouds
}

\author{
F. Toschi ${ }^{1,2}$, P. Capuzzi ${ }^{3}$, S. Succi ${ }^{1,3}$, P. Vignolo ${ }^{3}$, and M. P. \\ $\mathrm{Tosi}^{3}$ \\ ${ }^{1}$ Istituto per le Applicazioni del Calcolo, CNR, Viale del Policlinico 137, I-00161 \\ Roma, Italy \\ 2 INFM, Unità di "Tor Vergata", Via della Ricerca Scientifica 1, I-00133 Roma, Italy \\ 3 NEST-INFM and Classe di Scienze, Scuola Normale Superiore, I-56126 Pisa, Italy \\ E-mail: toschi@iac.cnr.it
}

\begin{abstract}
We study the transition from the collisionless to the hydrodynamic regime in a two-component spin-polarized mixture of ${ }^{40} \mathrm{~K}$ atoms by exciting its dipolar oscillation modes inside harmonic traps. The time evolution of the mixture is described by the Vlasov-Landau equations and numerically solved with a fully three-dimensional concurrent code. We observe a master/slave behaviour of the oscillation frequencies depending on the dipolar mode that is excited. Regardless of the initial conditions, the transition to hydrodynamics is found to shift to lower values of the collision rate as temperature decreases.
\end{abstract}

Submitted to: J. Phys. B: At. Mol. Opt. Phys.

PACS numbers: $05.30 . \mathrm{Fk}, 71.10-\mathrm{w}$ 


\section{Introduction}

Several experiments on cooling two spin-polarized states of a Fermi gas have proven that this setup is an important tool for investigating the collisional properties of such a quantum system [1. The JILA group has recently performed experiments in which the collisionality of a ${ }^{40} \mathrm{~K}$ mixture is tuned by either varying the atomic density [1, 2] or the off-resonant value of the inter-species scattering length [3, 4]. Also the temperature of the gas has been used to drive its collisionality and the importance of Pauli blocking at very low temperatures has thereby been demonstrated [1]. The role of collisions has also been investigated in dipolar collective modes of a boson-fermion mixture [5] at temperatures in both the quantum and classical regimes. The behaviour of the collision rate inferred from these experiments plays a crucial role in the development of strategies to improve the evaporative cooling techniques. In addition, the collisionality also affects other observables such as the damping and diffusion processes in the quantum mixture. In Ref. 4] an anisotropic expansion of a cigar-shaped two-component Fermi gas has been observed. In contrast to the bosonic case, the anisotropy of the expansion at very low temperatures cannot be taken as a clear signature of fermionic superfluidity [6] and could be due to purely collisional effects.

While ergodic approximations on semiclassical Boltzmann equations have been used to describe the evaporative cooling process [7], a microscopic understanding of the transition towards hydrodynamics [1] or of the anisotropic expansion in the case of attractive interactions [8] needs a complete numerical study of the dynamics of the quantum gas in position and momentum space [9. In this paper we describe the strategy that we have used to solve numerically the Vlasov-Landau Equations (VLE) for an ultracold two-component fermion gas with a fully three-dimensional (3D) algorithm. The two fermionic fluids are treated by a particle-dynamics approach [10], accounting for mean-field interactions and for collisions between the two species. Since the Pauli principle causes a saturation of phase space at very low temperature, we have developed a locally adaptive importance-sampling technique which allows us to select the colliding particles in a way which is by several orders of magnitude faster than in standard Monte Carlo techniques. Furthermore, the control of the occupancy of the unitary cells of phase space and a suitable choice of computational parameters permit us to avoid Pauli inconsistencies during the dynamics.

The two fermionic gases are confined inside different harmonic traps. Regardless of

the initial perturbation exciting the dipolar modes, we observe that as temperature is lowered, even through most collisions become forbidden classically and by the Pauli principle, the few collisions that occur still suffice to drive the mixture from the collisionless to the hydrodynamic regime. This effect has already been predicted in our previous study [9], where the angular degree of freedom was taken into account via an effective weight in a $2 \mathrm{D}$ numerical code. The limitations of the $2 \mathrm{D}$ approach are illustrated in the present work by comparing the oscillation frequencies and the damping rate of the dipolar oscillations evaluated within the $2 \mathrm{D}$ approach with those obtained 
by means of the present 3D scheme. We also find that the choice of the initial state modifies the spectra of the dipolar modes. In particular, if the symmetry of the initial configuration is broken and one species is initially at rest, kicking by the other species will induce oscillations at the characteristic frequency of the latter.

The paper is organized as follows. In Sec. 2 we introduce the physical model and describe in detail the numerical method developed to solve it. In Sec. 3 we study the dipolar oscillations in various collisional regimes and compare the results with those obtained in our previous work. Concluding remarks are given in Sec. 4.

\section{The model and its solution in $3 \mathrm{D}$}

The two fermionic components in external potentials $V_{\text {ext }}^{(j)}(\boldsymbol{r})$ are described by the distribution functions $f^{(j)}(\boldsymbol{r}, \boldsymbol{p}, t)$ with $j=1$ or 2 . They obey the kinetic equations

$$
\partial_{t} f^{(j)}+\frac{\boldsymbol{p}}{m} \cdot \nabla_{\boldsymbol{r}} f^{(j)}-\nabla_{\boldsymbol{r}} U^{(j)} \cdot \nabla_{\boldsymbol{p}} f^{(j)}=C_{12}\left[f^{(j)}\right]
$$

where the mean-field effective potential is $U^{(j)}(\boldsymbol{r}, t) \equiv V_{\text {ext }}^{(j)}(\boldsymbol{r})+g n^{(\bar{j})}(\boldsymbol{r}, t)$ with $\bar{j}$ denoting the species different from $j$. Here we have set $\hbar=1, g=2 \pi a / m_{r}$ with $a$ being the $s$-wave scattering length between two atoms of different species and $m_{r}$ the reduced mass, and $n^{(j)}(\boldsymbol{r}, t)$ is the density obtained by integrating $f^{(j)}(\boldsymbol{r}, \boldsymbol{p}, t)$ over momentum.

Collisions between atoms of the same spin can be neglected at low temperature, so that in Eq. (11) the term $C_{12}$ involves only collisions between particles that are polarized in two different Zeeman states. We have:

$$
C_{12}\left[f^{(j)}\right] \equiv g^{2} \frac{2(2 \pi)^{4}}{V^{3}} \sum_{\boldsymbol{p}_{2}, \boldsymbol{p}_{3}, \boldsymbol{p}_{4}} \Delta_{\boldsymbol{p}} \Delta_{\varepsilon}\left[\bar{f}^{(j)} \bar{f}_{2}^{(\bar{j})} f_{3}^{(j)} f_{4}^{(\bar{j})}-f^{(j)} f_{2}^{(\bar{j})} \bar{f}_{3}^{(j)} \bar{f}_{4}^{(\bar{j})}\right]
$$

with $f^{(j)} \equiv f^{(j)}(\boldsymbol{r}, \boldsymbol{p}, t), \bar{f}^{(j)} \equiv 1-f^{(j)}, f_{i}^{(j)} \equiv f^{(j)}\left(\boldsymbol{r}, \boldsymbol{p}_{i}, t\right), \bar{f}_{i}^{(j)} \equiv 1-f_{i}^{(j)} . \quad V$ is the volume occupied by the gas and the factors $\Delta_{p}$ and $\Delta_{\varepsilon}$ are the usual delta functions accounting for conservation of momentum and energy, with the energies given by $p_{i}^{2} / 2 m_{j}+U^{(j)}(\boldsymbol{r}, t)$.

The equilibrium state of the mixture is given by the stationary solution of Eq. (1), i.e. by the local Fermi-Dirac distributions

$$
f^{(j)}(\boldsymbol{r}, \boldsymbol{p})=\left(e^{\beta\left(p^{2} / 2 m_{j}+U^{(j)}(\boldsymbol{r})-\mu_{j}\right)}+1\right)^{-1}
$$

at given temperature $T=1 / k_{B} \beta$, where $\mu_{j}$ is the chemical potential ensuring the normalization condition $\int f^{(j)}(\boldsymbol{r}, \boldsymbol{p}) d^{3} r d^{3} p / h^{3}=N_{j}$. The particle densities entering $U^{(j)}$ are to be determined self-consistently by integration over momenta. The details of this calculation have been previously given in Ref. [11.

\subsection{Numerical method}

The numerical solution of the coupled VLE in Eq. (II) is carried out inside a finite box of size $L_{x} \times L_{y} \times L_{z}$ and discretized with a spacing $\Delta x_{j}$. We describe each species 
in the box by means of a set of $N_{c}$ computational particles (cp) at phase-space points $\left\{\boldsymbol{r}_{i}, \boldsymbol{p}_{i}\right\}$ which are spread according to the distribution $f$. In this way each fermion is represented by $N_{q}=N_{c} / N_{j}>1$ "quarks".

The initial selection of the computational particles is made by direct Monte Carlo sampling of the equilibrium distributions. Moreover, since collisions are very sensitive to the statistical nature of the colliding particles, we enforce the Pauli principle in each phase-space volume of size $h^{3}$ by resampling the computational particles that would otherwise exceed the allowed number of quarks $N_{q}$ in that cell. This further control is needed since we are dealing with a finite-size sample of Fermi-Dirac distributions.

The solution of Eqs. (11) proceeds in two steps: (i) the Lagrangian evolution of each distribution in phase space due to mean-field and external forces, and (ii) the occurrence of binary collisions among particles of the two species à la Boltzmann. Once the initial state is prepared, the first stage of the evolution is carried out by means of a secondorder symplectic integrator [12, 13, and we update the position and velocity of each particle $i$ according to the scheme

$$
\left\{\begin{array}{ll}
\tilde{\boldsymbol{r}}_{i} & =\boldsymbol{r}_{i}(t)+\frac{1}{2} \Delta t \boldsymbol{v}_{i}(t), \\
\boldsymbol{v}_{i}(t+\Delta t) & =\boldsymbol{v}_{i}(t)+m^{-1} \Delta t \boldsymbol{F}\left(\tilde{\boldsymbol{r}}_{i}\right) \\
\boldsymbol{r}_{i}(t+\Delta t) & =\tilde{\boldsymbol{r}}_{i}+\frac{1}{2} \Delta t \boldsymbol{v}_{i}(t+\Delta t)
\end{array} .\right.
$$

Here $\boldsymbol{F}(\boldsymbol{r})$ is the mean-field force $\boldsymbol{F}(\boldsymbol{r})=-\nabla U^{(j)}(\boldsymbol{r})$ with $j=1$ or 2 . To compute the mean-field forces we first calculate the average density at a given cell by counting the number of particles inside the cell. The density is then numerically differentiated to obtain the forces at the grid points. The forces so calculated are finally linearly interpolated to the positions of the particles.

It is worthwhile remembering that Liouvillean evolutions are incompressible, so that a distribution function satisfying the Pauli principle at a given time will satisfy the same constraint afterwards. This is an exact property whose numerical counterpart is checked during the course of the simulation.

The second step of the solution involves binary collisions. At variance from the Lagrangian evolution these are tracked on a coarser mesh of spacing of the order of the de Broglie wavelength (a "Pauli cell"). In this way we introduce a temperaturedependent scale which imposes a length scale on which the particles are effectively indistinguishable. This can be seen as an extension of the standard Boltzmann approach. Furthermore, collisions in different Pauli cells can be handled independently due to the locality of Eq. (2).

Our method allows us to count the number of collisions that occur step-by-step both classically and quantum-mechanically. In each Pauli cell we add up the classical probability of collisions between every pair of particles and store the pairs whose probability is greater than a locally adapted threshold. This reduces the running time and the storage needs of the code by several orders of magnitude, and at the same time the threshold is adjusted in order to guarantee a correct supply of pairs at each time step. The quantum features of the mixture are included in the last step of the 
collision algorithm where the Pauli suppression in Eq. (2) is applied. For every classically colliding pair we calculate the blocking terms $\left(1-f^{(j)}\right)$ by analyzing the occupancy of the momentum states in the Pauli cell. We have explicitly verified that this procedure yields the correct Pauli-suppressed fraction of collisions for a weakly interacting gas at least down to $T \sim 0.2 T_{F}[14$.

We turn now to the discussion of the choice of computational parameters which allows us a reliable implementation of the Lagrangian evolution. During the dynamics two unphysical processes may occur: (i) the presence of an excess of quarks in saturated phase-space cells, and (ii) a broadening of the atomic cloud inside the trap. No cell of volume $h^{3}$ contains more than $N_{q}$ quarks for each species if the initial state has been accurately prepared. In this case, the inconsistent location of quarks takes place after we move the particles. Indeed, both problems (i) and (ii) are related to the calculation of the accelerations induced by mean-field forces. As already mentioned, these forces are constructed first on the simulation grid and then interpolated to the actual position of each particle. Therefore, an accurate description requires small spacings $\Delta x_{j}$ and a large number of computational particles per cell.

Aiming at understanding the impact on the results of the different choices for the values of $N_{q}$ and $L_{j} / \Delta x_{j}$ we have studied the axial motion of two interacting Fermi clouds for several values of these parameters. Hereafter we shall consider magnetically trapped ${ }^{40} \mathrm{~K}$ atoms that are equally shared in two different Zeeman states $\left(m_{F}=9 / 2\right.$ and $\left.m_{F}=7 / 2\right)$ and confined in isotropic harmonic traps with slightly different frequencies $\left(\omega_{9 / 2}=2 \pi \times 19.8 \mathrm{~s}^{-1}\right.$ and $\left.\omega_{7 / 2}=2 \pi \times 17.46 \mathrm{~s}^{-1}\right)$. For this test case we take the total number of particles $N=200$, the mutual scattering length $a=3 \times 10^{4}$ Bohr radii (a strongly collisional regime), and $T=0.3 T_{F}$. Both clouds are suddenly displaced from their equilibrium positions along the axial direction and start oscillating. We then analyze the evolution of the mixture for a total time $t_{f i n} \simeq 50 \omega_{9 / 2}^{-1}(\sim 8$ complete oscillations) and from the value of the centre-of-mass position $z_{j}(t)$ we extract the oscillation frequencies $\omega_{j}$ of the two components and the average damping rate $\bar{\gamma}$. The details of this procedure will be given in Sec. 3. In addition we also compute the axial spreads of the density profiles $D_{j}=\left\langle\Delta z_{j}\right\rangle_{t_{f i n}} /\left\langle\Delta z_{j}\right\rangle_{t_{0}}$ and the quantum collision rate $\Gamma_{q}$ by direct counting of the total collisions per particle, and monitor the number $\mathcal{N}$ of computational particles located in forbidden phase-space cells. The latter shall be called Pauli inconsistencies. For the system parameters here considered, we find that the oscillation frequencies do not vary considerably as $N_{q}$ and $L_{j} / \Delta x_{j}$ are varied. The dependence of the other observables is summarized in Table 1 .

The inconsistencies disappear by increasing the number of grid points, while a large number of computational particles per cell is required to prevent statistical noise from producing spurious diffusion of the particles. The average damping rate $\bar{\gamma}$ is sensitive to the spreading of the clouds, but seems to be less affected by the presence of few inconsistencies. On the other hand the collisional rate $\Gamma_{q}$ depends more strongly on both factors.

In summary, this test case has given us enough information to select appropriate 
Table 1. The maximum relative value $\tilde{\mathcal{N}}=\left(\mathcal{N} / N_{c}\right)_{\max }$ of particles in excess per time step, the maximum spread $D=\max \left\{D_{9 / 2}, D_{7 / 2}\right\}$ of the clouds in the axial direction, the collision rate $\Gamma_{q}$ (in units of $1 / \mathrm{s}$ ), and the average damping rate $\bar{\gamma}$ (in units of $1 / \mathrm{s})$ as functions of the number of quarks $N_{q}$ and of grid points $L_{j} / \Delta x_{j}$. The data correspond to the two-component Fermi gas at $T=0.3 T_{F}$ with a total number of particles $N=200$ and an inter-species scattering length $a=3 \times 10^{4} a_{0}\left(a_{0}\right.$ being the Bohr radius).

\begin{tabular}{lcccc}
\hline & $\tilde{\mathcal{N}}$ & $D$ & $\Gamma_{q}(1 / \mathrm{s})$ & $\bar{\gamma}(1 / \mathrm{s})$ \\
\hline$L_{j} / \Delta x_{j}=100$ & & & & \\
$N_{q}=20$ & $4 \times 10^{-3}$ & 1.40 & 21.10 & 1.26 \\
$N_{q}=50$ & $7 \times 10^{-3}$ & 1.20 & 23.05 & 1.33 \\
$N_{q}=200$ & $6 \times 10^{-2}$ & 1.00 & 21.60 & 1.07 \\
$L_{j} / \Delta x_{j}=200$ & & & & \\
$N_{q}=200$ & 0 & 1.40 & 21.80 & 1.50 \\
$N_{q}=400$ & 0 & 1.25 & 23.50 & 1.30 \\
$N_{q}=1600$ & 0 & 1.00 & 29.10 & 1.06 \\
\hline
\end{tabular}

parameters for reliable calculations. In the following numerical experiments we shall choose the most suitable values of $N_{q}$ and $L_{j} / \Delta x_{j}$ in order to ensure $D_{j} \simeq 1$ and a negligible $\mathcal{N}$, while keeping the computing time within reasonable limits.

\section{Dipolar oscillations}

The quantum features of the mixture are most conveniently displayed by its collective modes, which in turn can be investigated by analyzing the dynamical response of the system under distortions of the equilibrium state. This can be done quite straightforwardly in actual experiments where the cloverleaf setup is used [1, 2, 3, 4]: a bias magnetic field displaces the centre of the trap, while a Stern-Gerlach separation of the two Zeeman states could be obtained by adding a magnetic field gradient [1, 15].

In the following we analyze the dipolar modes of the mixture at varying scattering length, as can be done e.g. by exploiting Feshbach resonances [3], and focus on two specific types of initial conditions: one in which both components start oscillating with the same amplitude (in-phase oscillations) and the other in which only the $m_{F}=9 / 2$ species is initially displaced (kicked oscillations).

\subsection{In-phase oscillations}

The equilibrium fermionic density profiles for $2 \times 10^{4}$ particles are first created with the traps centred at $\boldsymbol{r}=(0,0,1) a_{h o}$. At $t=0$ we let the confinements return to the position $\boldsymbol{r}=0$ and hence the atoms start oscillating. In the collisionless regime the natural oscillation frequencies of the two species are different and correspond to trap frequencies renormalized by mean-field interactions, whereas the hydrodynamic regime is characterized by a common oscillation frequency of the two clouds, whose motions 


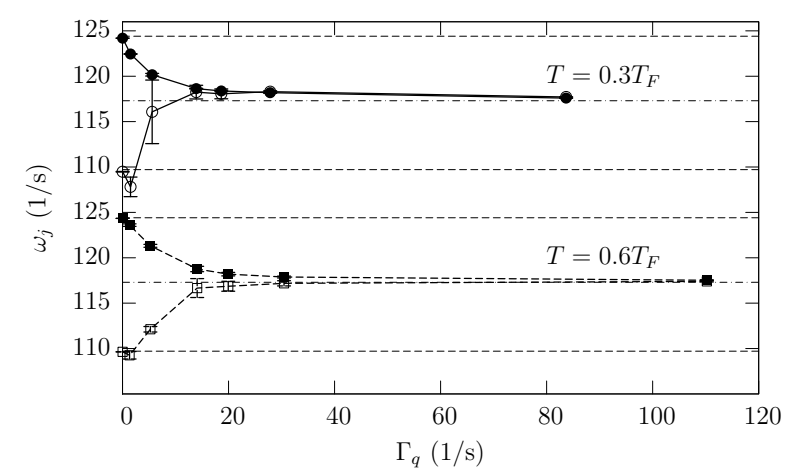

(a)

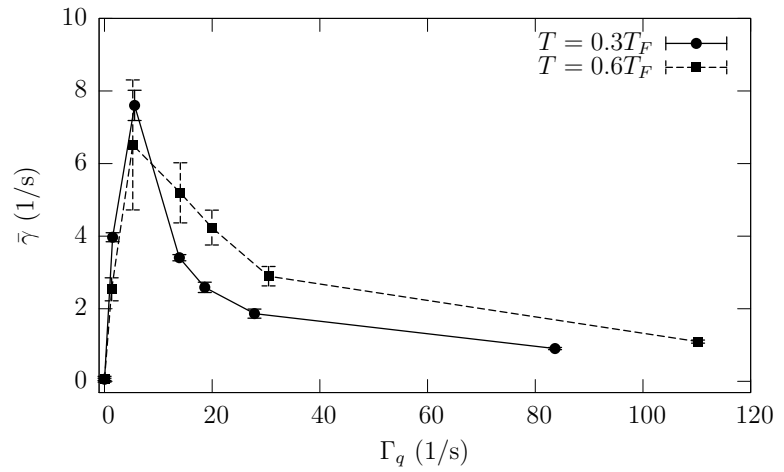

(b)

Figure 1. Transition from the collisionless to the collisional regime in a ${ }^{40} \mathrm{~K}$ mixture with $N_{7 / 2}=N_{9 / 2}=10^{4}$ atoms. (a) Oscillation frequencies (in units of $1 / \mathrm{s}$ ) as a function of the collision rate $\Gamma_{q}$ (in units of $1 / \mathrm{s}$ ). The circles correspond to $T=0.3 T_{F}$ while the squares to $T=0.6 T_{F}$; filled symbols mark the $m_{F}=9 / 2$ species and the empty ones the $m_{F}=7 / 2$ species. The dash-dotted lines indicate the location of $\omega_{H D}$ while the horizontal dashed lines indicate the bare trap frequencies. (b) Average damping rate $\bar{\gamma}$ (in units of $1 / \mathrm{s}$ ) as a function of $\Gamma_{q}$.

have been locked by frequent collisions between their particles. In this limit classical kinetic theory predicts that the common frequency is $\omega_{H D}=\sqrt{\left(\omega_{9 / 2}^{2}+\omega_{7 / 2}^{2}\right) / 2}$, which in our case gives $\omega_{H D} \simeq 117.3 \mathrm{~s}^{-1}$. This is in agreement with our numerical results as is shown in Fig. 1(a), where the in-phase dipolar frequencies are plotted as functions of the collision rate $\Gamma_{q}$.

In evaluating the oscillation frequencies and damping rates we have assumed that the dynamics is well described by an exponentially damped harmonic motion and fitted each centre-of-mass position $z_{j}(t)$ with a function $A_{j} \cos \left(\omega_{j} t+\phi_{j}\right) \exp \left(-\gamma_{j} t\right)$. We calculate the average damping rate as $\bar{\gamma}=\left(\gamma_{9 / 2}+\gamma_{7 / 2}\right) / 2$ and estimate the uncertainty of the result by analyzing the dependence of the fit parameters on the fitting range. This uncertainty is reported in the figures as error bars. The intermediate regime is characterized by a strong damping and larger uncertainties in the single-mode frequencies, which destroy the coherence of the oscillations.

As an illustration of the various dynamical regimes we show in Fig. 2 the evolution of the centre of mass of the two species for three values of $\Gamma_{q}$ at $T=0.3 T_{F}$. For $2 \pi \Gamma_{q} \omega_{j}^{-1} \ll 1$ the two clouds oscillate independently without appreciable damping, whereas as $2 \pi \Gamma_{q} \omega_{j}^{-1}$ approaches unity the two centres of mass start moving together but still incoherently and therefore their oscillations are damped. At higher collisionality $\left(2 \pi \Gamma_{q} \omega_{j}^{-1} \gg 1\right)$ the two species are locked and oscillate at the same frequency without damping.

From Figures 1(a) and 1(b) it is clear that the transition towards the hydrodynamic regime occurs for lower values of $\Gamma_{q}$ at $T=0.3 T_{F}$ than at $T=0.6 T_{F}$. This phenomenon 


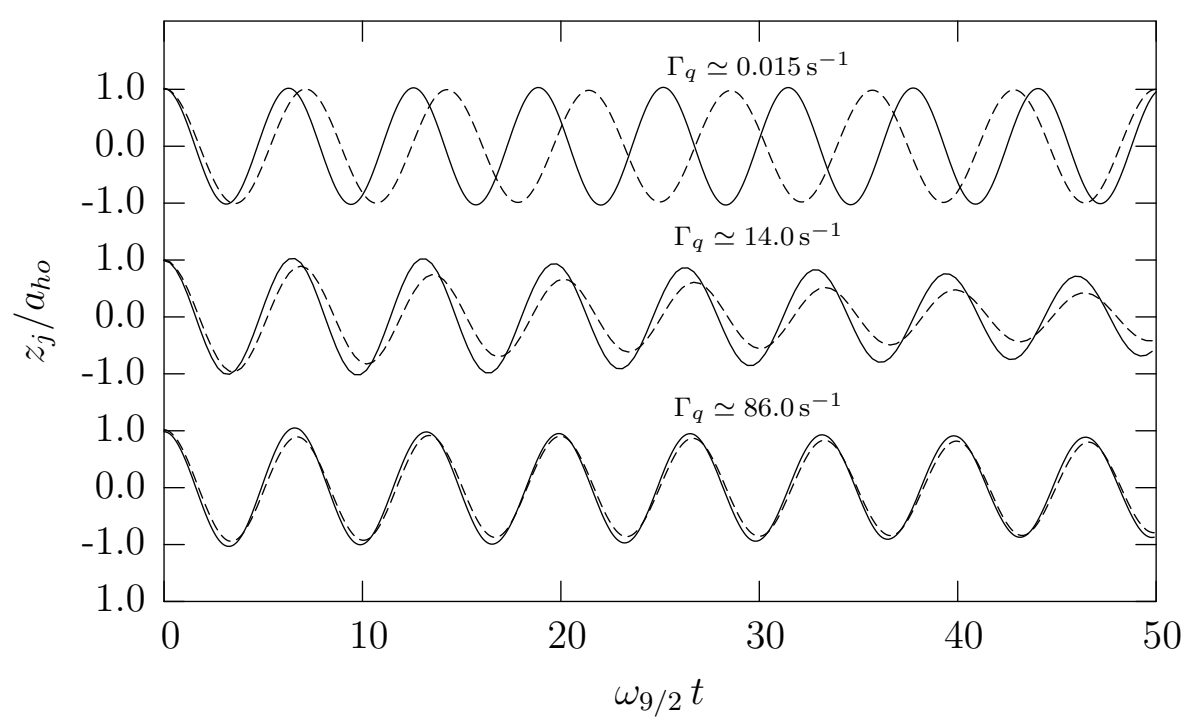

Figure 2. Centre-of-mass position $z_{j}(t)$ (in units of the oscillator length $a_{h o}=$ $\sqrt{\hbar / m \omega_{9 / 2}}$ ) as functions of $\omega_{9 / 2} t$ for a mixture of ${ }^{40} \mathrm{~K}$ atoms at $T=0.3 T_{F}$. From top to bottom, the indicated values of the collisional rate correspond to $a=150 a_{0}$ (collisionless regime), $a=5.0 \times 10^{3} a_{0}$ (intermediate regime), and $a=1.5 \times 10^{4} a_{0}$ (hydrodynamic regime), in units of the Bohr radius $a_{0}$.

can be attributed to collisions which involve particles in a narrower energy range around the Fermi level as the temperature decreases [9]. However, the results presented here are only in qualitative agreement with those obtained in Refs. [9. In fact, the $2 \mathrm{D}$ approach overestimates the collision rate and the damping rate. This is shown in Fig. B where the oscillation frequencies are compared with those obtained by using the $2 \mathrm{D}$ code.

\subsection{Kicked oscillations}

We next excite the dipolar mode with a different initial configuration, by taking the trap centre at $(0,0,0)$ for the $m_{F}=7 / 2$ component and at $(0,0,1) a_{h o}$ for the $m_{F}=9 / 2$ component. The confinement for the $m_{F}=9 / 2$ component is then shifted to $z=0$ and only its atoms start oscillating. If the two clouds did not interact, the $m_{F}=9 / 2$ component would oscillate at its bare frequency and the centre-of-mass of the other species would not move. Collisionality drives both clouds into oscillation.

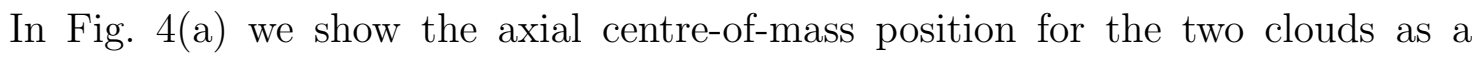
function of time for four values of the collision rate $\Gamma_{q}$. At variance from what was found in the in-phase case, a single-mode model cannot describe the oscillations since even in the collisionless region the $m_{F}=7 / 2$ motion has a strong frequency component induced by the $m_{F}=9 / 2$ motion. This can be seen from the corresponding Fourier transform $\left|\hat{z}_{j}(\omega)\right|$ of the centre-of-mass positions in the various regimes, which are shown in Fig. [4(b). In the case of small collisionality (the two top curves in Fig. 四) the $m_{F}=9 / 2$ component acts as a drive on the $m_{F}=7 / 2$ component but is not strongly affected by collisions. 


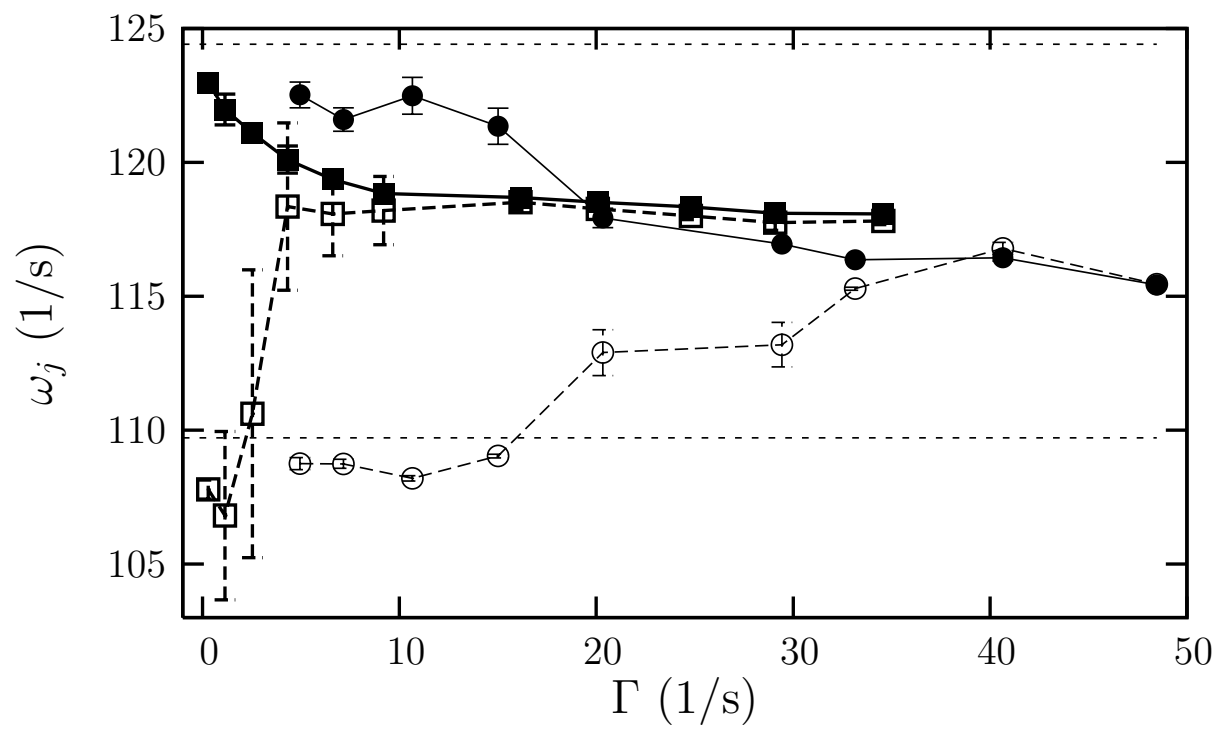

Figure 3. Comparison of the oscillation frequencies $\omega_{j}$ (in units of $1 / \mathrm{s}$ ) obtained with the present 3D approach (squares) with those of an effective 2D system (circles) for a ${ }^{40} \mathrm{~K}$ mixture with $N_{9 / 2}=N_{7 / 2}=100$ particles at $T=0.3 T_{F}$. The empty symbols correspond to the $m_{F}=7 / 2$ species and the filled ones to the $m_{F}=9 / 2$ one.

The corresponding $z_{j}(t)$ trajectories are drawn in Fig. 4(a). For low collisionality (top and second row in Fig. (4), a beating between the two peaks in the $m_{F}=7 / 2$ spectrum is visible in the $z_{7 / 2}(t)$ signal. At higher collisionality the two clouds get locked and oscillate at a single frequency, which is intermediate between the two trap frequencies (see also the spectrum in Fig $4(b))$ ).

\section{Summary and concluding remarks}

We have studied the transition from the collisionless to the hydrodynamic regime in a ${ }^{40} \mathrm{~K}$ mixture at temperatures where the effects of Pauli blocking are noticeable. The VlasovLandau equations of two interacting fermionic fluids are solved numerically by means of a fully three-dimensional particle-dynamics approach incorporating mean-field and collisional interactions. The implementation of a locally-adaptive importance sampling technique during the collisional step has allowed us to obtain a method which is very efficient at low temperature, for realistic number of particles and in a wide range of collisional strength. By exploiting this code we investigate the dipolar collective modes and analyze the oscillation frequencies and damping rates of the system for various initial configurations. We find that the transition to hydrodynamics is shifted to lower values of the collision rate as temperature decreases.

As a future application, this procedure shall permit us to perform numerical experiments on cooling and expansion dynamics. These studies will help characterize the mixture in the intermediate regime where neither purely collisionless nor collisional approaches are appropriate. In addition the extension to highly interacting systems 


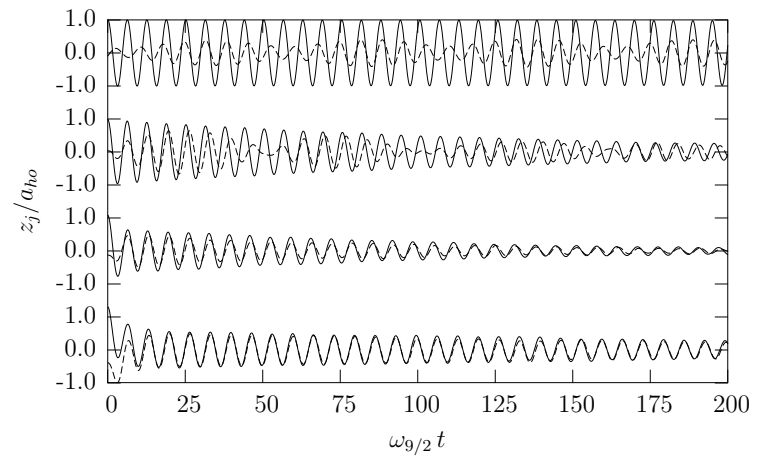

(a)

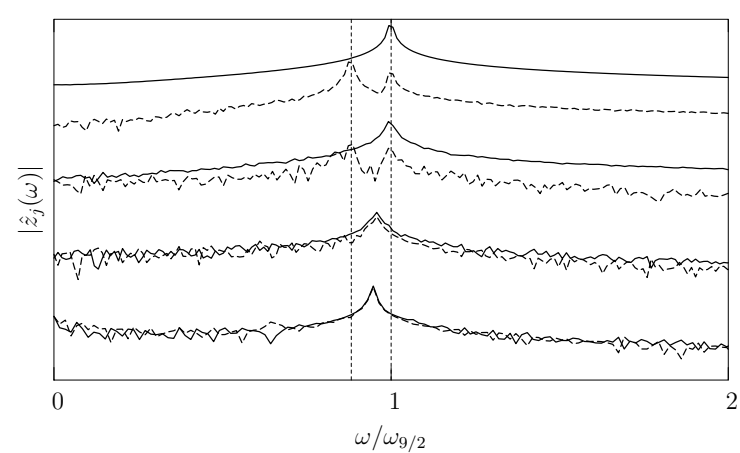

(b)

Figure 4. Kicked oscillations of the $m_{F}=9 / 2$ component (full lines) and the $m_{F}=7 / 2$ component (dashed lines) of a ${ }^{40} \mathrm{~K}$ mixture at $T=0.3 T_{F}$ with $N=2 \times 10^{4}$ atoms. (a) Centre-of-mass positions $z_{j}(t)$ (in units of the oscillator length $a_{h o}$ ) as a function of $\omega_{9 / 2} t$ : from top to bottom the data correspond to $a=150 a_{0}$ (collisionless regime), $a=1.5 \times 10^{3} a_{0}$ (intermediate regime), $a=5.0 \times 10^{3} a_{0}$ (intermediate regime approaching collisional), and $a=1.5 \times 10^{4} a_{0}$ (hydrodynamic regime). The amplitude of $z_{7 / 2}(t)$ has been rescaled by a factor 10 and 5 in the top and second row, respectively. (b) Corresponding Fourier transform $\left|\hat{z}_{j}(\omega)\right|$ (in arbitrary units and log scale) as a function of the frequency $\omega$ (in units of $\omega_{9 / 2}$ ). The vertical dashed lines indicate the location of the bare trap frequencies.

in the so-called unitary limit of collisions [16] shall provide more physical insight into recent experiments on attractive mixtures of fermionic atoms [17. Efforts to pursue these further studies are currently underway and will be reported elsewhere.

\section{Acknowledgments}

This work has been partially supported by INFM under the PRA-Photonmatter Programme.

\section{References}

[1] Gensemer S D and Jin D S 2001 Phys. Rev. Lett. 87173201.

[2] DeMarco B and Jin D S 2002 Phys. Rev. Lett. 88040405.

[3] Loftus T, Regal C A, Ticknor C, Bohn J L and Jin D S 2002 Phys. Rev. Lett. 88173201.

[4] Regal C A and Jin D S 2003 Phys. Rev. Lett. 90230404.

[5] Ferlaino F, Brecha R J, Hannaford P, Riboli F, Roati G, Modugno G and Inguscio M 2003 J. Opt. B: Quantum Semiclass. Opt. 5 S3.

[6] Gupta S, Hadzibabic Z, Anglin J R and Ketterle W 2003 cond-mat /0307088.

[7] Geist W and Kennedy T A B 2002 Phys. Rev. A 65063617.

[8] Regal C A, Ticknor C, Bohn J L and Jin D S 2003 Phys. Rev. Lett. 0532012003.

[9] Toschi F, Vignolo P, Succi S and Tosi M P 2003 Phys. Rev. A 67041605.

[10] Bird G 1994 Molecular Gas Dynamics and the Direct Simulation of Gas Flows Oxford University Press Oxford. 
[11] Amoruso M, Meccoli I, Minguzzi A and Tosi M P 1999 Euro. Phys. J. D 7441.

[12] Nettesheim P, Bornemann F A, Schmidt B and Schütte C 1996 Chem. Phys. Lett. 256581.

[13] Jackson B and Zaremba E 2002 Phys. Rev. A 66033606.

[14] Succi S, Toschi F, Capuzzi P, Vignolo P and Tosi M P 2004 Phil. Trans. R. Soc. . Submitted.

[15] Stamper-Kurn D M, Miesner H J, Inouye S, Andrews M R and Ketterle W 1998 Phys. Rev. Lett. 81500.

[16] Gehm M E, Hemmer S L, O'Hara K M and Thomas J E 2003 Phys. Rev. A 6811603.

[17] O'Hara K M, Hemmer S L, Gehm M E, Granade S R and Thomas J E 2002 Science 2982179. 